\title{
A NOTE ON THE ASYMPTOTIC BEHAVIOUR OF THE SUM OF PRINCIPAL RADII OF CURVATURE ON NONCOMPACT COMPLETE HYPERSURFACES
}

\author{
V. I. OLIKER
}

\begin{abstract}
It is shown that under certain hypotheses the following conjecture is correct: on a noncompact complete hypersurface in Euclidean space the two conditions below cannot hold simultaneously:

(i) the sum of principal radii of curvature is bounded;

(ii) the support function is uniformly continuous.
\end{abstract}

I. The main result. Let $\mathcal{T}$ be a noncompact $C^{3}$ hypersurface in Euclidean space $E^{m+1}(m>2)$, and $r(u)$ the position vector of $\mathcal{T} ; u=\left\{u^{i}\right\}, i=$ $1, \ldots, m$, are the local coordinates on $\mathcal{T}$. Assume that $\mathcal{T}$ is equipped with the metric induced from $E^{m+1}$. Suppose also that $\mathcal{T}$ is orientable and oriented. If it is not so then we pass to the universal covering of $\mathcal{T}$ and then work on that covering. Under such circumstances there exists on $\mathcal{T}$ a $C^{2}$ vector field of unit normals, and one may consider the Gauss map $\gamma: \mathcal{T} \rightarrow \Sigma$, where $\Sigma$ is the hypersphere of unit radius in $E^{m+1}$ centered at the origin. By $\mathcal{T}^{*}$ we denote a set on $\Sigma$ which contains the limits of all converging sequences of the form $\gamma\left(p_{k}\right)$ where $p_{k}$ is a sequence of points on $\mathcal{T}$ unbounded in the metric of $\mathcal{T}$.

In this note we shall study the asymptotic behaviour of $\mathcal{T}$. For that reason our further assumptions are related to an "infinite" part of $\mathcal{T}$. Assume that $\mathcal{T}^{*} \neq \varnothing$. Then we call a "leaving domain" any submanifold $\mathcal{T}^{\prime}$ of $\mathcal{T}$ with the following properties:

(a) there exists an open domain $B$ on $\Sigma$ with a boundary of class $C^{k}$ $(k>2)$ such that $B \cap \mathcal{T}^{*}=\varnothing, \partial B \cap \mathcal{T}^{*} \neq \varnothing$ and $\gamma$ is a diffeomorphism mapping $\mathcal{T}^{\prime}$ onto $B$;

(b) for any sequence of points $n_{k} \in B$ converging to a point from $\mathcal{T}^{*}$ the sequence $\gamma^{-1}\left(n_{k}\right)$ is an unbounded sequence on $\sigma^{\prime}$.

We also say that a leaving domain $\mathcal{T}^{\prime}$ is asymptotically regular if the support function $h(u)=(r(u), n(u)), n \in \mathcal{T}^{\prime}$, transplanted via $\gamma$ on $B$ can be extended to $B+\partial B$ as a continuous function and its restriction $\varphi(u)=$ $\left.h(u)\right|_{\partial B}$ is a $C^{1, \lambda}$ function, $0<\lambda<1$. Here $($, ) means, as usual, the inner product in $E^{m+1}$.

Received by the editors December 5, 1978 and, in revised form, February 14, 1979.

AMS (MOS) subject classifications (1970). Primary 53C40. 
Geometrically, asymptotic regularity means that for each connected component of $\partial B \cap \mathcal{T}^{\prime *}$ all asymptotes to the corresponding part of $\mathcal{T}^{\prime}$ lie in a family of hyperplanes defined by the function $\varphi(u)$.

Finally, we note that, like the function $h(u)$, any function defined on a leaving domain can be transplanted via $\gamma$ to $B$.

In what follows, by an $L^{p}$ norm, $p \geqslant 1$, of a function on $\mathcal{T}^{\prime}$ we understand the $L^{p}$ norm with respect to the volume element of the standard metric of $\Sigma$.

Now we are ready to state:

THEOREM. Let $\mathcal{T}$ be as above and $\mathcal{T}^{*} \neq \varnothing$. Suppose that there exists a leaving domain $\mathcal{T}^{\prime} \subset \mathcal{T}$. Then the following two conditions cannot hold simultaneously on $\mathcal{T}^{\prime}$ (hence on $\mathcal{T}$ ):

(i) the sum of the principal radii of curvature $R(u), u \in \sigma^{\prime}$, has a finite $L^{m+\delta}$ norm for some positive $\delta$;

(ii) $\mathcal{T}^{\prime}$ is asymptotically regular.

COROLlARY. If a $C^{3}$ noncompact hypersurface $\mathcal{T}$ contains an asymptotically regular leaving domain $\mathcal{T}^{\prime}$, then

$$
\sup _{u \in \mathscr{T}^{\prime}}|R(u)|=\infty
$$

It is not difficult to give examples illustrating the theorem. For instance, a catenoid in $E^{3}$ is a surface on which $R(u) \equiv 0$ but obviously no leaving domain of it is asymptotically regular. Moreover, for the catenoid both conditions defining asymptotic regularity are violated. In general, the above theorem asserts that a minimal surface cannot have a leaving domain which is asymptotically regular.

A branch of hyperbola when rotated about one of its asymptotes gives an example of a surface which has an asymptotically regular leaving domain. This can be easily seen if we note that the subset of $\mathcal{T}^{*}$ corresponding to the "horn" of this surface is an equator of $\Sigma$, and as a domain $B$ we can take a narrow strip along this equator. In this case all asymptotes to the corresponding part of the surface coincide with the asymptote of the original hyperbola. Placing the origin of coordinate system in $E^{3}$ on this asymptote we can make $\varphi(u) \equiv 0$. The corollary implies then that $R(u)$ cannot be bounded on such a surface.

II. The proof. (1) At first we note that if $\mathcal{T}^{\prime}$ is a leaving domain of $\mathcal{T}$ then $\sigma^{\prime}$ can be recovered from its support function as

$$
r(u)=\operatorname{grad} h(u)+h(u) n(u), \quad u \in B,
$$

where the gradient is taken in the standard metric of $\Sigma$. This time $u$ is a point in $B \subset \Sigma$. The representation (1) is possible because $\gamma$ is a $C^{2}$ diffeomorphism on $\mathcal{T}^{\prime}$ and that implies that the Gauss curvature $K \neq 0$ on $\mathcal{J}^{\prime}$. Under such circumstances the existence of representation (1) is shown, for example, by Hartman and Wintner [3]. Note, however, that if $\mathcal{J}^{\prime}$ was originally of class 
$C^{3}$, (1) gives in general only a $C^{2}$ representation. At the same time the support function remains of class $C^{3}$ in $B$.

(2) Here we show that a leaving domain is unbounded in $E^{m+1}$. For that we observe at first that for any connected subdomain $\tilde{B} \subset B$ such that $\partial \tilde{B} \cap \mathcal{T}^{*} \neq \varnothing$, the hypersurface $\mathcal{T}_{1}=\{r=r(u), u \in \tilde{B}\}$ will have the property (b) of a leaving domain. Select $\tilde{B}$ sufficiently small so that there could be found a point $c \in \Sigma$ such that both $c$ itself and the point $\bar{c}$ symmetric to $c$ with respect to the center of $\Sigma$ do not lie in $\mathcal{T}_{1}^{*}$. This is possible since the Gauss map $\gamma$ is a diffeomorphism on $\mathscr{T}_{1}$. Now take a path $l$ in $\tilde{B}$ with an endpoint $q$ in $\mathscr{T}_{1}^{*}$. Because of the way the diameter $c \bar{c}$ was chosen it becomes apparent that near the point $q$ the angles between the normals to the tangent hyperplanes to $\mathcal{T}_{1}$ along $l$ and the diameter $c \bar{c}$ are bounded away from zero. Therefore, a height function on $\mathcal{T}_{1}$ in the direction $c \bar{c}$ has no critical points and $q$ is not a critical point at infinity. Since $\mathscr{T}_{1}$ is a leaving domain, the latter implies that $\mathcal{T}_{1}$ is unbounded in the direction $c \bar{c}$.

The above argument is actually a special case of a more general proof of unboundedness of complete submanifolds in Euclidean space (see Verner [5]).

(3) Because of (2) we have

$$
|r(u)| \underset{u \rightarrow q}{\rightarrow} \infty, \quad q \in \mathcal{T}_{1}^{*} \subset \mathcal{T}^{\prime *}, \quad u \in B .
$$

In view of (1) this may occur only if either $|\operatorname{grad} h|$ or $|h|$, or both, becomes unbounded when $u \rightarrow q$. However, if $\sigma^{\prime}$ is asymptotically regular then $h$ is continuous in $B+\partial B$, and therefore it is the $|\operatorname{grad} h|$ which must be unbounded. We want to show that it is impossible if $R(u) \in L^{m+\delta}(B)$ for some $\delta>0$.

The functions $R(u)$ and $h(u)$ are related by the Weingarten equation (see, for example, [3])

$$
\Delta_{2} h(u)+m h(u)=R(u), \quad u \in B,
$$

where $\Delta_{2}$ is the Laplace operator on $\Sigma$. As has already been mentioned the function $h(u) \in C^{3}(B)$; hence $R(u) \in C^{1}(B)$. Suppose that $R(u) \in C^{1}(B) \cap$ $L^{p}(B)$ where we write for brevity $p=m+\delta$. Since $\mathcal{T}^{\prime}$ is asymptotically regular, $\left.h(u)\right|_{\partial B}=\varphi(u) \in C^{1, \lambda}(\partial B)$. Denote by $f(u)$ the solution of the following boundary value problem

$$
\begin{aligned}
\Delta_{2} f(u) & =0 \quad \text { when } u \in B, \\
f(u) & =\varphi(u) \quad \text { when } u \in \partial B .
\end{aligned}
$$

By our assumptions the boundary $\partial B$ is not empty and it is of class $C^{2}$. Under such circumstances the solution $f(u)$ exists, is unique, and is of class $C^{2}(B) \cap C^{1, \lambda}(B+\partial B)$ (see [2, p. 102]).

Put $H(u)=h(u)-f(u)$. Subsituting this in (3) we obtain

$$
\Delta_{2} H(u)=R(u)-m h(u), \quad u \in B .
$$

Also, $H(u)=0$ when $u \in \partial B$. Now, since $R(u)-m h(u) \in L^{p}(B)$ with $p>m$ $>2$, it follows that $H(u)$ belongs to the Sobolev space $W_{0}^{2,2}(B)$ (see [2, 
Theorems 8.9, p. 175, and 8.12, p. 176]). Moreover, the latter together with the hypothesis $p>m$ imply that $H(u) \in C^{1, \mu}(B+\partial B$ ), where $\mu=1-m / p$ (see [4, Theorem 15.1, p. 203]).

Since $h(u)=f(u)+H(u)$, and $f(u) \in C^{1, \lambda}(B+\partial B)$, the above means that $h(u) \in C^{1, l}(B+\partial B)$, where $l=\min \{\lambda, \mu\}$. Therefore $|\operatorname{grad} h|$ is bounded in $B+\partial B$. Thus we arrive at a contradiction with (2). The theorem is proved.

The corollary is an immediate consequence of the theorem.

III. Remarks. (1) The concept of a leaving domain has been introduced by Cohn-Vossen [1] in a much more general form. For two-dimensional complete surfaces in $E^{3}$ with univalent Gauss map the leaving domains and the limiting sets of their Gauss images were studied by Verner [6].

(2) Our result is probably true under milder conditions defining the asymptotic behavior of leaving domains. For example, it seems plausible that the condition requiring the support function of an asymptotically regular leaving domain to be of class $C^{1, \lambda}$ on the boundary of its Gauss image can be dropped. However, in this case part (3) of the proof would need an approach different from ours.

Acknowledgement. The author wishes to thank the referee for valuable comments.

\section{BIBLIOGRAPHY}

1. S. Cohn-Vossen, Kürzeste Wege and Totalkrümmung auf Flächen, Compositio Math. 2 (1935), 69-133.

2. D. Gilbarg and N. S. Trudinger, Elliptic partial differential equations of second order, Springer-Verlag, Berlin, 1977.

3. P. Hartman and A. Wintner, On the third fundamental form of a surface, Amer. J. Math. 75 (1953), no. 2, 298-334.

4. O. A. Ladyzhenskaya and N. N. Uraltseva, Linear and quasi-linear elliptic equations, Academic Press, New York, 1968.

5. A. L. Verner, The unlimitedness of a hyperbolic horn in the Euclidean space, Sibirsk. Mat. $\grave{Z}$. 11 (1970), 20-29; English transl., Siberian Math. J. 11 (1970), 15-21.

6. On the extrinsic geometry of elementary complete surfaces with nompositive curvature. I, II, Mat. Sb. 74 (116) (1967), 218-240; 75 (117) (1968), 112-139; English transl., Math. USSR Sb. 3 (1967), 205-224; 4 (1968), 99-123.

Division of Mathematical Sciences, University of Iowa, Iowa City, Iowa 52242 